\title{
A multi-center trial of LGE-MRI of the left atrium
}

\author{
Eugene Kholmovski ${ }^{1,2^{*}}$, Kavitha Damal ${ }^{1}$, Nathan S Burgon ${ }^{1}$, Sathya Vijayakumar ${ }^{1,2}$, Chankevin Tek ${ }^{1}$, \\ Anya G Mihaylova', Nassir F Marrouche ${ }^{1}$ \\ From 16th Annual SCMR Scientific Sessions \\ San Francisco, CA, USA. 31 January - 3 February 2013
}

\section{Background}

Atrial fibrillation (AF) is the most common rhythm disturbance. Radio-frequency ablation (RFA) of the left atrium (LA) is effective for drug refractory AF patients. Outcome of RFA procedure depends on the degree of pre-ablation LA fibrosis and amount of post-ablation scar. Late gadolinium enhancement (LGE) imaging can detect fibrosis and visualize scar. However, only few centers with advanced expertise in cardiac MR (CMR) have demonstrated successful LGE of LA. Multi-center study was initiated to study reproducibility of LGE of LA.

\section{Methods}

11 clinical centers with different degrees of CMR expertise and typical MRI hardware have participated in this study. Customized pulse sequences and imaging protocols for LGE of LA were installed on 13 Siemens scanners at the centers: $1.5 \mathrm{~T}$ - 4 Avanto, 2 Espree, 1 Sonata, 1 Symphony; 3 T - 3 Verio, 2 Trio. 6 centers used 1.5T scanners, 4 centers used 3T scanners and 1 center used both 1.5 and 3T. 3 centers used specialized cardiac coils; the others used standard body and spine coils. The participating centers followed their regular protocol for CMR contrast injection (Table 1). MRI technologists in most centers underwent 1-2 days training in LA imaging.

$370 \mathrm{AF}$ patients underwent LGE-MRI within 30 days prior to RFA. LGE-MRI was repeated for $270 \mathrm{AF}$ patients at least 3 months after RFA to quantify post-ablation scar. Quality of LGE images was scored by two experienced blinded readers: good - 2 , fair - 1 , poor - 0 . In cases of score discrepancy, consensus was achieved by the readers. Poor quality images were not clinically useable. Good and fair quality images can be used for fibrosis and scar quantification. Poor quality images and supporting data (heart rhythm and rate, navigator signal, etc.) were analyzed to find the main reason for scan failure.

\section{Results}

The results of image analysis are summarized in Table 2 . Quality was better for pre-ablation scans performed on 3T scanners than on 1.5T $(\mathrm{p}<0.01)$. Post-ablation scans consistently have better quality than pre-ablation scans ( $<<0.02$ ). $13.0 \%$ of pre and $9.3 \%$ of post-ablation scans was graded as poor. In $62 \%$ of the cases MRI technologist error was the main reason for poor quality. Typical errors were wrong inversion time, wrong phase-encoding direction, partial coverage of LA, navigator prescription error, poor ECG signal, and error in the main frequency adjustment (only at $3 \mathrm{~T}$ ). In $31 \%$ cases, poor quality was patient related: significant arrhythmia, very irregular respiration, heart rate $>120 \mathrm{bpm}$, patient motion, and extreme obesity. Artifacts in parallel imaging reconstruction on Espree were responsible for poor quality in $7 \%$ cases.

\section{Conclusions}

The multi-center trial demonstrates that it is possible to consistently acquire clinically useable LGE of LA using customized sequences and imaging protocols in centers without advanced CMR expertise utilizing typical MRI hardware and contrast agents. Better training in LA imaging for MRI technologists, may further improve image quality of LGE of LA.

Table 1 Type of contrast agent and dosage in the participating sites

\begin{tabular}{ccc}
\hline Contrast agent & The number of centers & Dose $(\mathrm{mmol} / \mathrm{kg})$ \\
\hline Dotarem & 1 & 0.2 \\
\hline Gadovist & 3 & $0.15,0.15,0.2$ \\
\hline Magnevist & $4^{*}$ & $0.1,0.2,0.2,0.2$ \\
\hline Multihance & $3^{*}$ & 0.1 \\
\hline Omniscan & 1 & 0.2 \\
\hline
\end{tabular}

*- one center switched from Magnevist to Mutihance during the study. 
Table 2 Image quality of pre- and post-ablation LGE-MRI of the left atrium

\begin{tabular}{|c|c|c|c|c|c|c|}
\hline & & Pre-ablation & & & Post-ablation & \\
\hline Scanner & $1.5 \mathrm{~T}$ & $3 T$ & $1.5 \& 3 T$ & $1.5 \mathrm{~T}$ & $3 T$ & $1.5 \& 3 T$ \\
\hline \# scans & 161 & 209 & 370 & 101 & 169 & 270 \\
\hline Good & $39.8 \%$ & $53.1 \%$ & $47.3 \%$ & $53.5 \%$ & $59.8 \%$ & $57.4 \%$ \\
\hline Fair & $43.5 \%$ & $36.8 \%$ & $39.7 \%$ & $31.7 \%$ & $34.3 \%$ & $33.3 \%$ \\
\hline Poor & $16.7 \%$ & $10.1 \%$ & $13.0 \%$ & $14.8 \%$ & $5.9 \%$ & $9.3 \%$ \\
\hline Score & $1.230 \pm 0.718$ & $1.431 \pm 0.669$ & $1.343 \pm 0.697$ & $1.386 \pm 0.734$ & $1.539 \pm 0.608$ & $1.482 \pm 0.661$ \\
\hline
\end{tabular}

\section{Author details}

'CARMA Center, University of Utah, Salt Lake City, UT, USA. 'UCAIR,

Department of Radiology, University of Utah, Salt Lake City, UT, USA.

Published: 30 January 2013

doi:10.1186/1532-429X-15-S1-0111

Cite this article as: Kholmovski et al: A multi-center trial of LGE-MRI of

the left atrium. Journal of Cardiovascular Magnetic Resonance 201315

(Suppl 1):0111.

Submit your next manuscript to BioMed Central and take full advantage of:

- Convenient online submission

- Thorough peer review

- No space constraints or color figure charges

- Immediate publication on acceptance

- Inclusion in PubMed, CAS, Scopus and Google Scholar

- Research which is freely available for redistribution

Submit your manuscript at www.biomedcentral.com/submit
C Biomed Central 